Amit Bar-Or, MD

Rogier Q. Hintzen, MD, $\mathrm{PhD}$

Russell C. Dale, MD, $\mathrm{PhD}$

Kevin Rostasy, MD

Wolfgang Brück, MD

Tanuja Chitnis, MD

Correspondence to

Dr. Bar-Or:

amit.bar-or@mcgill.ca

\title{
Immunopathophysiology of pediatric CNS inflammatory demyelinating diseases
}

\section{ABSTRACT}

Elucidating pathophysiologic mechanisms underlying the spectrum of pediatric-onset CNS demyelinating diseases, particularly those that may distinguish multiple sclerosis (MS) from other entities, promises to both improve diagnostics and guide more-informed therapeutic decisions. Observations that pediatric- and adult-onset MS share the same genetic and environmental risk factors support the view that these conditions represent essentially the same illness manifesting at different ages. Nonetheless, special consideration must be given when CNS inflammation manifests in early life, at a time when multiple organs (including immune and nervous systems) are actively maturing. CSF analysis in pediatric-onset MS points to chronic CNS inflammation, supported by observations from limited pathologic material available for study. Emerging results implicate abnormalities in both effector and regulatory T cell subsets, and potentially immune senescence, in children with MS. Although CNS-directed antibodies (including antibodies recognizing myelin antigens; Kir4.1) can be documented in pediatric-onset MS, their pathophysiologic significance (as in adults) remains unclear. This is in contrast to the presence of serum and/or CSF antibodies recognizing aquaporin-4, which, when measured using validated cell-based assays, supports the diagnosis of a neuromyelitis optica spectrum disorder, distinct from MS. Presence of anti-myelin oligodendrocyte glycoprotein antibodies documented with similar cell-based assays may also be associated with pathophysiologically distinct disease phenotypes in children. The substantial impact of pediatriconset MS on normal brain development and function underscores the importance of elucidating both the immunobiology and neurobiology of disease. Ongoing efforts are aimed at developing and validating biological measures that define pathophysiologically distinct monophasic and chronic forms of pediatric CNS demyelination. Neurology ${ }^{\circledR}$ 2016;87 (Suppl 2):S12-S19

\section{GLOSSARY}

ADEM = acute disseminated encephalomyelitis; AQP4 = aquaporin-4; BBB = blood-brain barrier; $\mathbf{C B A}=$ cell-based assay; EDSS = Expanded Disability Status Scale; MBP = myelin basic protein; MOG = myelin oligodendrocyte glycoprotein; MS = multiple sclerosis; NMO = neuromyelitis optica; NMOSD = NMO spectrum disorder; OCB = oligoclonal band; ON = optic neuritis; OPC = oligodendrocyte precursor cell; $\mathbf{T M}=$ transverse myelitis.

There is presently limited insight into the pathophysiologic mechanisms underlying childhoodonset inflammatory demyelinating diseases. Because these disorders occur in the context of the developing immune and nervous systems, understanding the implications to both disease mechanisms and efficacy and safety profiles of potential therapeutics will help guide optimal management approaches for these children and adolescents. Elucidating the biology of pediatric-onset multiple sclerosis (MS) may provide key insights into earliest targets and processes involved in disease pathogenesis as well as into the broader spectrum of CNS demyelinating disease.

In this article, we focus on the pathophysiology of pediatric-onset MS, occasionally commenting on other conditions in the pediatric inflammatory demyelinating disease spectrum, including optic neuritis $(\mathrm{ON})$, transverse myelitis (TM), neuromyelitis optica (NMO) or NMO spectrum disorders (NMOSDs), and acute disseminated encephalomyelitis (ADEM), which are discussed in

From the Neuroimmunology Unit and Experimental Therapeutics Program (A.B.-O.), Montreal Neurological Institute, McGill University, Montreal, Quebec, Canada; Department of Neurology (R.Q.H.), MS Centre ErasMS, Neurology, Erasmus MC, Rotterdam, the Netherlands; Neuroimmunology Group (R.C.D.), Institute for Neuroscience and Muscle Research, the Children's Hospital at Westmead, University of Sydney, Australia; Department of Pediatric Neurology (K.R.), Witten/Herdecke University, Children's Hospital Datteln, Datteln, Germany; Department of Neuropathology (W.B.), University Medical Center Göttingen, Georg-August-University, Göttingen, Germany; and Partners Pediatric MS Center (T.C.), Massachusetts General Hospital, Harvard Medical School, Boston, MA.

Go to Neurology.org for full disclosures. Funding information and disclosures deemed relevant by the authors, if any, are provided at the end of the article. 
greater detail elsewhere in this supplement. We consider general implications of inflammatory CNS disease manifesting in the context of less mature immune and nervous systems. We provide an overview of genetic and environmental risk factors implicated in the development of pediatric-onset MS and discuss insights from the relatively limited body of work to date examining brain pathology, CSF profiles, CNS-reactive antibodies, and cellular immune responses in pediatric-onset MS and related disorders. In doing so, we highlight both challenges as well as opportunities in the evolving field of pediatric inflammatory demyelinating diseases.

GENERAL ASPECTS OF CNS INFLAMMATORY DEMYELINATING DISEASES MANIFESTING IN THE YOUNG As described in detail in this supplement, the same genetic and environmental risk factors implicated in adult-onset MS have been shown to contribute to MS risk in pediatric-onset MS (figure 1), supporting the view that similar pathophysiologic mechanisms underlie development of MS whether clinical onset is in early life or later. In addition, children with MS do not appear to manifest particular comorbidities that might point to a greater genetic burden-although the relatively small numbers available for study and the possibility that young individuals may not have manifested comorbid illnesses yet (and would thus be inappropriately classified) limit interpretation of such studies. ${ }^{1}$ However, in contrast to adults with MS, both the immune and nervous systems are undergoing active development in children and adolescents developing MS or other inflammatory CNS diseases. Although much of an individual's adaptive immune system (comprising $\mathrm{T}$ cells and $\mathrm{B}$ cells) is established by early life, these immune repertoires must continue to expand and mature, such as through thymic release of newly educated $\mathrm{T}$ cells and bone marrow-derived $\mathrm{B}$ cells and plasma cells. In contrast, innate immune responses are quite intact even in a neonate. Children experience a large number of new immune challenges, including initial exposures to viruses, bacteria, parasites and fungi, during the first year of life and again during early school age. In many regions children are given vaccinations to further induce or boost particular immune responses. Recurrent infections not only stimulate the immune system but also affect the integrity of the blood-brain barrier (BBB), which in turn may increase exposure of the CNS to peripheral immune mediators. A number of infectious, parainfectious, and postinfectious inflammatory CNS syndromes occur more commonly in children than in adults. The known female predilection for MS is not seen in the youngest children (in whom the F:M sex ratio is $1: 1$ ) and appears to emerge after age 11 or so (likely reflecting puberty), ${ }^{1}$ suggesting that both age and sex may impact an individual's early life risk of developing MS. Although "immaturity of the immune system" has been proposed as a reason that MS is less common in children than adults, the immune system in children is overall quite mature. The fact that other inflammatory conditions, both CNS-directed (e.g., myelin oligodendrocyte glycoprotein (MOG) antibodyassociated disease, ADEM) and systemic (e.g., type 1 diabetes, certain rheumatologic disorders), appear more commonly in children than in adults makes this "immaturity" argument less tenable (reviewed in ref. 1 and 2).

How inflammatory responses may affect the stilldeveloping pediatric CNS is an important and relatively poorly explored field. The early-life CNS harbors more immature cells, including oligodendrocyte precursor cells (OPCs; the potential remyelinating cells), than the adult CNS. In this regard, OPC survival and differentiation have been shown to be affected by distinct immune cell subsets implicated in MS pathophysiology, both directly and through modulation of other glial cells such as astrocytes. ${ }^{3}$ A common theory is that the CNS of children may be more "plastic" than that of adults and that it may be better able to compensate for injury. The observation that development of substantial motor disability is relatively uncommon in children with MS in spite of considerably more active inflammatory disease may reflect improved repair mechanisms in children, although it may also reflect less loss of reserve than adults with (on average) longerstanding biological disease. It is also noteworthy that, in spite of good motor recovery, children with MS can be significantly affected in terms of cognitive functions, school performance, and socialization; therefore, the notion of greater plasticity and repair in this population should not be overestimated.

The lesser maturity of both immune and nervous systems in children with MS also has important therapeutic implications because these systems may be differentially affected by approved and emerging MS therapies compared to adults. Of particular importance is the potential impact of different immune interventions on the relatively active thymus and bone marrow, on the establishment of mature and diverse immune repertoires, and on immune surveillance including in the CNS. The fact that some immune processes (such as response to injury and repair) are actually beneficial should be considered. For the reasons outlined above, caution should be exercised in extrapolating insights and 

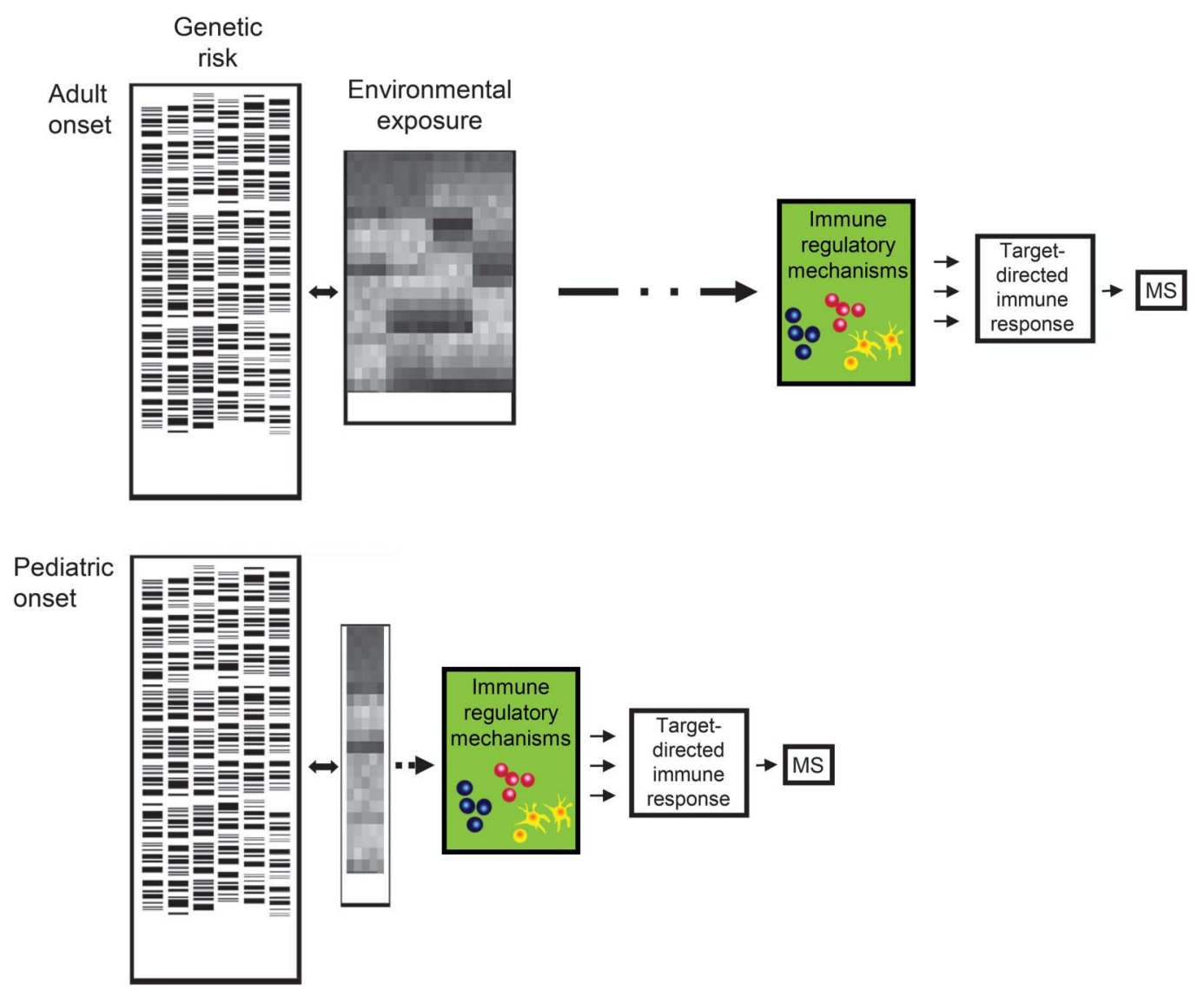

Genetic and environmental factors interact over time to confer risk of developing multiple sclerosis (MS). Patients with pediatric-onset MS come to clinical attention at a time that is closer on average to biological disease onset. This would be associated with a narrower range of environmental exposures acting on genetic susceptibility, providing a unique window to investigate early targets and mechanisms involved in the regulation of CNS-directed immune responses.

experience from adults to children with CNS inflammatory disease.

PATHOLOGY OF MS Most descriptions of MS pathology are derived from postmortem studies in progressive, long-standing MS cases. The characteristic pathologic hallmark of MS is the confluent, sharply demarcated white matter lesion showing demyelination, inflammation, gliosis, and relative axonal preservation (figure $2 \mathrm{~A}$ and reviewed in ref. 4). Studies have demonstrated that demyelination also affects the gray matter, especially the cortical gray matter. Here, 3 different lesion types can be distinguished: the most frequent lesion type is the subpial lesion, followed by leukocortical and purely intracortical lesions. The deep gray matter is also substantially involved in the demyelinating process. Remyelination can be observed in MS lesions and may be particularly evident in early disease stages ${ }^{5}$ and in the cortex compared to the white matter. ${ }^{6}$

More-detailed insight into the pathology of early disease stages is derived from studies of biopsy tissue from early relapsing-remitting MS cases. These studies have shown heterogeneity of the MS lesions, ${ }^{5-7}$ more extensive remyelination in early disease stages, ${ }^{5}$ and a higher degree of acute damage to axons in younger patients and those with shorter disease duration. ${ }^{8}$

Studies on the pathology of MS in pediatric patients are extremely rare. A recent systematic analysis of pediatric MS white matter lesions analyzed the extent of acute damage to axons in relation to the amount of inflammation within the lesions and the disability of the patients. ${ }^{9}$ The findings showed a 50\% higher degree of acute damage to axons in the children with MS than the adults with MS. In addition, the degree of acute damage to axons correlated negatively with the patients' age at the time of either biopsy or autopsy. ${ }^{9}$ The prepubertal age group in this study had the highest degree of damage to axons. Inflammation within the lesions also showed a clear age dependency. Although there was no difference in $\mathrm{T}$ cell numbers between pediatric and adult MS cases, macrophage/microglia numbers were highest in prepubertal MS lesions followed by 


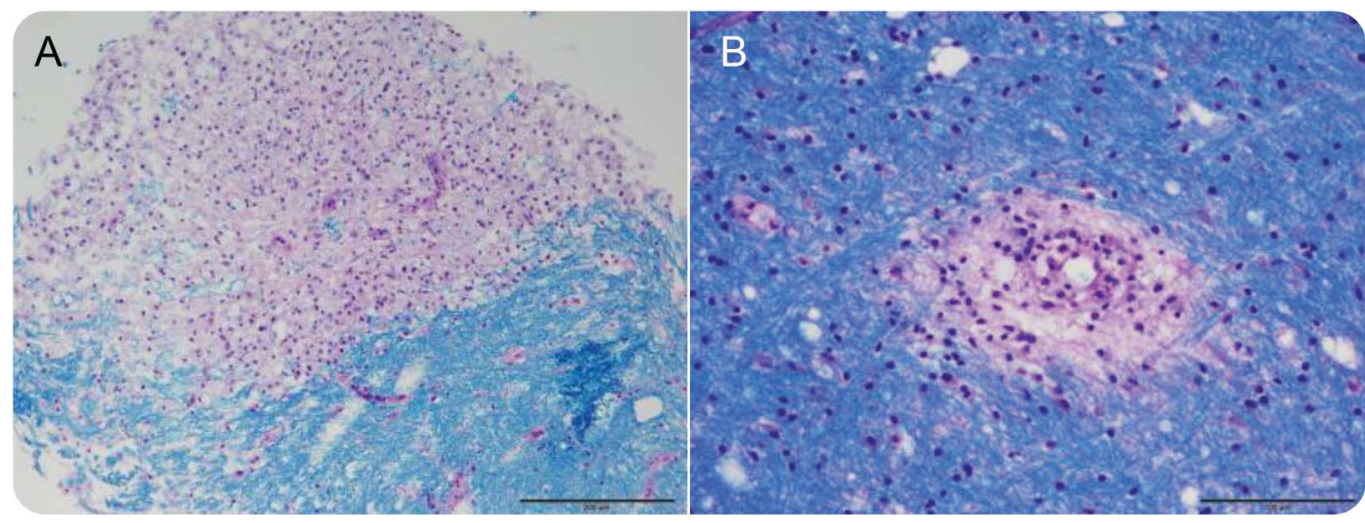

(A) Typical multiple sclerosis (MS) lesion showing a confluent demyelinated plaque with sharp border to the periplaque white matter ( $\times 100$, Luxol fast blue-periodic acid-Schiff [LFB-PAS] myelin stain). (B) Acute disseminated encephalomyelitis (ADEM) lesion showing limited perivascular inflammation and demyelination ( $\times 200$, LFB-PAS myelin stain).

postpubertal MS and adult MS lesions, indicating that the innate immune reaction in pediatric MS may be significantly more extensive than that in adult MS. ${ }^{9}$ This was also related to a higher MRI burden of T2-hyperintense lesions in these patients. ${ }^{9}$ Moreover, a clear correlation existed between the degree of acute damage to axons within lesions and the Expanded Disability Status Scale (EDSS) score at the time of biopsy or autopsy. Remyelination was also observed in the lesions; however, a detailed quantification was not performed. In summary, these first published data on the pathology of pediatric MS highlight the substantial degree of acute damage to axons within inflammatory demyelinating lesions of children compared to such lesions in adults. ${ }^{9}$

The major differential diagnoses in the pathology of pediatric MS include ADEM and NMO. ADEM is characterized histopathologically by small perivascular demyelinating lesions (figure 2B). The perivascular demyelinated area is infiltrated by numerous foamy macrophages, a few lymphocytes $\left(\mathrm{CD}^{-}\right.$and CD8-positive T cells, B cells, and plasma cells), and very rarely granulocytes. In contrast to MS lesions, demyelination is generally limited to perivascular areas with inflammatory infiltrates and the large confluent demyelinated lesions typical for MS do not form. ${ }^{10,11}$ In very acute ADEM lesions, perivascular complement deposits can be found, possibly pointing toward an antibody-/complement-mediated pathogenesis. ${ }^{12,13}$ Although NMO was once considered a variant of MS, the discovery of the autoantibody marker NMO-IgG directed against aquaporin-4 $(\mathrm{AQP} 4)^{14}$ has since established NMO as a pathophysiologically distinct entity. AQP4 is a water channel located on astrocytes and concentrated on their foot processes at the BBB. In contrast to MS lesions,
NMO lesions show dystrophic astrocytes associated with perivascular deposition of IgG and complement and a striking loss of $\mathrm{AQP} 4$ extending beyond the area of demyelination. ${ }^{15,16}$ In general, NMO lesions are more destructive than MS lesions, leading to extensive loss of axons and oligodendrocytes.

CSF PROFILES CSF analysis is an important tool in considering the differential diagnosis of a child with an acute inflammatory demyelinating event and can offer important insights into the pathophysiology, particularly in children with a chronic demyelinating disorder. Common features of MS in both children and adults are the intrathecal humoral immune responses characterized by the presence of CSFrestricted $\operatorname{IgG}$ oligoclonal bands (OCBs) and local synthesis of a limited antibody repertoire including antibodies against measles, rubella, and varicellazoster virus-features that are not entirely specific to MS and may be absent early in the disease course, particularly in children under age $10 .{ }^{17-20}$ IgG OCBs are more common in children with MS than in those with ADEM or other diseases mimicking MS. ${ }^{17-19,21}$ Presence of $\mathrm{IgG}$ OCBs combined with an abnormal MRI was associated with a $>26$-fold hazard ratio for developing MS in children presenting with incident $\mathrm{ON} .^{22}$

CSF IgM OCBs can also be found in a subset of patients with pediatric- and adult-onset MS. In adults their presence has been associated with more active/ aggressive disease, ${ }^{23}$ but such an association has not been established in pediatric MS. ${ }^{24}$

Of note, CSF exhibits age-related changes independent of MS disease mechanisms, particularly around puberty. Reiber et al. ${ }^{20}$ showed that the amount of intrathecal IgG doubles during puberty because of an increase in the albumin quotient, which 
represents normal physiologic growth. Measuring the IgG index (not just IgG levels) should help avoid false interpretation of IgG synthesis rates in children and adolescents with MS. ${ }^{18,20}$ Children with prepubertal MS appear to have a slightly higher total CSF white cell count combined with a higher proportion of neutrophils than postpubertal children, ${ }^{18}$ although these values are still lower than those in their adult counterparts. $^{20}$ This may be due to differences in the developing immune system or reflect age-related MS-specific immune responses. With new techniques evolving, such as immunophenotyping of CSF B lymphocyte subpopulations, cell-based assays (CBAs), and measurement of proteins such as glial fibrillary acidic protein (a potential marker of astrocytic damage), the CSF profile of pediatric MS and other acquired demyelinating diseases could be studied in more detail with potential to establish biological markers that may affect diagnosis and early treatment decisions.

EFFECTOR AND REGULATORY T CELLS IN PEDIATRIC MS A simple model of the immune pathogenesis of MS can be conceptualized based on results from studies of immune responses in adults with MS, insights from use of experimental therapies that target particular immune responses, and complementary data from animal model studies. ${ }^{25,26}$ An early step is thought to involve peripheral activation of $\mathrm{CD}^{+} \mathrm{T}$ cells in response to some stimulating antigen, during which the profile of molecules including costimulatory signals and cytokines influences activated $\mathrm{T}$ cell responses and their capacity to interact with and transmigrate across the BBB. ${ }^{26}$ This transmigration involves adhesion, chemoattraction, and active infiltration of the activated immune cells into the CNS thought to contribute to perivascular inflammatory injury of the CNS. ${ }^{26}$ The process by which the injury may cause additional CNS antigens to be exposed, thereby resulting in the recruitment of $\mathrm{T}$ cells with specificities for additional CNS antigens, has been called "epitope spreading," which may also serve to propagate a chronic immune response. ${ }^{25,26}$ The initial antigenic targets in MS are unknown; the "molecular mimicry" hypothesis postulates this to be an infectious antigen, triggering $\mathrm{T}$ cell responses that are cross-reactive with CNS antigen. Compact myelin antigens such as myelin basic protein (MBP) and proteolipid protein, as well as MOG, have been traditionally considered leading candidates, largely based on the experience from the commonly used animal model experimental autoimmune encephalomyelitis. An exploratory CSF proteomic analysis comparing a small number of children with MS to children with monophasic disease failed to detect differences in compact myelin antigens while surprisingly implicating the axoglial apparatus (the small region where the oligodendrocyte myelin membrane attaches to the axonal node of Ranvier) as a potential target of early injury in pediatric MS. ${ }^{27}$

A number of abnormalities in $\mathrm{T}$ cell phenotype and function have been reported in adults with MS (reviewed in ref. 25 and 26), whereas relatively limited cellular immunology data are available in children. An early study suggested that children with early MS exhibited abnormally heightened circulating $\mathrm{T}$ cell responses to $\mathrm{CNS}$ autoantigens, although so did children with remote CNS injury, highlighting the challenge of distinguishing immune responses that contribute to injury from those that may develop as a response to injury. ${ }^{28} \mathrm{~A}$ subsequent study assessed responses of $\mathrm{T}$ cells of both adult and pediatric patients with MS to MBP and $\mathrm{MOG}^{29}$ and found that both groups mounted preferential and similar responses to particular antigenic epitopes, including MBP 83-102, 139-153, and 146-162 and MOG $1-26,38-60$, and 63-87.29 The authors noted that $\mathrm{T}$ cells of both children and adults with MS mounted limited responses to fetal MBP. A significant number of children on immunomodulatory treatments were included in these 2 studies, which may have influenced results. Two groups have since evaluated $\mathrm{T}$ cell responses in pediatric MS. In one study, $\mathrm{CD} 4^{+} \mathrm{T}$ cell responses to a composite of 7 myelin peptides were increased in a group of untreated pediatric patients with MS compared to adults with MS as well as healthy control children. ${ }^{30}$ Children with MS also exhibited higher frequencies of proliferating memory $\mathrm{CD}^{+} \mathrm{T}$ cells and higher levels of interleukin-17 secretion in response to myelin peptides than healthy children, suggesting that this $\mathrm{T}$ cell population may be relevant to pathogenesis of pediatric $\mathrm{MS}^{30}$ Another group found an increased proportion of memory cells and fewer recent thymic emigrants (particularly regulatory $\mathrm{T}$ cells) in children with MS compared to healthy children, suggesting early immune senescence in patients with pediatric MS. ${ }^{31}$ This study also found that the suppressive function of Foxp3 regulatory cells was impaired in patients with pediatric MS. The extent to which peripheral immune measures may (or may not) reflect the immune profile within the pediatric MS CNS remains to be elucidated.

CNS-DIRECTED ANTIBODIES IN PEDIATRIC DEMYELINATING DISEASE A growing number of CNS-reactive autoantibodies are emerging as useful diagnostic markers in the context of CNS inflammatory diseases, including pediatric demyelinating disorders. The method used to measure the CNS-autoreactive antibodies is an important consideration. For autoantibodies that bind to the extracellular domains of proteins such as AQP4 (in $\mathrm{NMO}^{14}$ ) and MOG, ${ }^{32}$ CBAs have emerged 
as the preferred approach, as these present the antigen in its conformational state at the cell surface, which is likely to be more representative of the in vivo interaction. ${ }^{33}$ In contrast, antibody assays that alter proteins (by unraveling them) or expose antigens or linear epitopes that are not present at the cell surface are less likely to measure antibodies that are pathophysiologically relevant in vivo. It remains unknown whether antibodies are primary contributors to pediatric CNS demyelinating disease immunopathogenesis, modify pathogenesis, or merely represent secondary epiphenomenon of immune activation. The classic autoantibody-mediated CNS demyelinating disease is $\mathrm{NMO}$, in which presence of the AQP4 targeting Ig in serum or CSF is associated with a typical perivascular staining pattern reflecting antibody binding to the foot processes of astrocytes around blood vessels. ${ }^{34}$ The anti-AQP4 antibody is thought to exert direct pathogenic functions (both complement- and Fc receptor-mediated), resulting in the hallmark autoimmune astrocytopathic picture distinguishing NMO from MS pathology.

Anti-MOG antibodies occur more frequently in children than in adults and have been described (using different assays which, as noted above, may affect findings) in pediatric patients with a variety of autoimmune demyelinating disease phenotypes, including those of ADEM, MS, ${ }^{32,35-37}$ relapsing ADEM, relapsing $\mathrm{ON},{ }^{38,39}$ relapsing $\mathrm{ADEM}-\mathrm{ON},{ }^{40}$ and relapsing ON-longitudinally extensive TM (NMO-like). ${ }^{36,37}$

The normal function of MOG, which resides at the cell surface of oligodendrocytes, is not well understood. There is a dominant epitope involved in MOG IgG binding on the extracellular domain ${ }^{13}$ and evidence of MOG IgG pathogenicity including alteration of oligodendrocyte cytoskeleton, ${ }^{38}$ as well as implication in both complement-dependent and cell-based cytotoxicity. ${ }^{35,41,42}$ A recent pathology report demonstrated antibody and complement deposition in an adult case of anti-MOG antibody-associated demyelination. ${ }^{43}$ Compared to AQP4 IgG-associated disease, CBA seropositivity for MOG IgG appears to be associated with less severe disease, including better resolution and less likelihood to relapse, ${ }^{44}$ and may also be associated with a non-MS disease phenotype. ${ }^{45}$

A serum antibody binding to KIR4.1 (the ATPsensitive inward rectifying potassium channel found primarily on glial cells) has been reported in $47 \%$ of adults with MS as well as in a subset of children with acute CNS demyelination. ${ }^{46,47}$ Presence of this antibody was not associated with particular clinical features and its significance is uncertain at this time. Studies that have not replicated the original report ${ }^{48,49}$ underscore the challenge of assay differences and the value of direct assay comparisons. ${ }^{33}$

One study using a custom antigen array of epitopes from candidate proteins including myelin antigens reported different $\operatorname{IgG}$ and $\operatorname{IgM}$ binding in serum of children with MS compared to those with ADEM. ${ }^{50}$ Another study applied an antigen array to samples obtained from children at time of presentation with incident episodes of CNS inflammatory demyelination as well as 3 months later. ${ }^{51}$ Children were subsequently diagnosed with either MS or monophasic disease. Although some differences were again noted in serum antibody patterns between children with MS and controls, of particular note was the observation that the range of CNS-directed antibodies detected in the children with MS increased substantially between the 2 time points, whereas the range decreased in children with monophasic disease. Although the pathogenic significance of particular serum antibodies detected using such antigen arrays is unclear, the general pattern and changes over time are consistent with a process of humoral "epitope spread," distinguishing the ongoing MS injury process from monophasic disease mechanisms.

CONCLUDING COMMENTS Information regarding the pathology and immunopathogenesis of pediatriconset MS and other early-life CNS inflammatory demyelinating conditions remains limited. Pediatricand adult-onset MS share genetic and environmental risk factors, suggesting that they represent essentially the same disease clinically manifesting at different ages rather than pathophysiologically distinct entities. Nonetheless, a number of differences exist. Children tend to experience more-frequent relapses and higher MRI lesion burden (more "inflammatory" disease, at least early on), have better motor recovery from acute relapses (with a slower progression on the EDSS scale), and experience an important impact on cognitive realms. Whether these differences reflect the lesser states of maturation of the immune and nervous systems in children, including differences in early-life CNS resiliency and/or repair capacities, is unknown. Studies of hypomyelinating conditions and leukodystrophies may provide additional insights into mechanisms of myelin injury and repair of particular relevance to pediatric-onset MS.52 Of concern are hints from imaging studies that progressive disease mechanisms are already in play at the earliest stage of the pediatric MS spectrum. ${ }^{53,54}$ Atrophy is present at the initial clinical presentation of children with MS, and their skull sizes appear on average smaller than controls, raising the question of how early the CNS injury process actually starts in these children. ${ }^{55}$ Therefore, patients with pediatric MS require treatments that would target both aberrant immune responses as well as the neurobiology of disease. Both current and future treatments must consider the impact of intervention on the still-developing immune and nervous systems. 


\section{AUTHOR CONTRIBUTIONS}

All authors contributed to manuscript concept and design. All authors provided critical revisions.

\section{STUDY FUNDING}

This supplement is made possible by funding from the MS Cure Fund, Danish MS Society, German MS Society, Italian MS Association, MS International Federation, MS Research Foundation (Netherlands), National MS Society (USA) and Swiss MS Society.

\section{DISCLOSURE}

A. Bar-Or has participated as a speaker at meetings sponsored by, has received consulting fees from, and/or has received grant support from Biogen Idec, Diogenix, Genentech, GSK, EMDSerono, Mitsubishi Tanaba, Novartis, Ono Pharma, Sanofi/Genzyme, Receptos, Roche, and Teva Neuroscience. A. Bar-Or serves on the editorial boards of Neurology and Cellular and Experimental Neuroimmunology. R. Hintzen has received honoraria for serving on advisory boards for Biogen Idec, Roche, and Sanofi. He participated in trials with Biogen Idec, Merck-Serono, Roche, Genzyme, and Novartis. He serves on the editorial board of Multiple Sclerosis and Related Disorders. R. Dale has received research funding from the National Health and Medical Research Council, MS Research Australia, Star Scientific Foundation, Pfizer Neuroscience, Tourette Syndrome Association, University of Sydney, and the Petre Foundation. R. Dale has received honoraria from Biogen Idec and Bristol-Myers Squibb as an invited speaker. K. Rostasy reports no disclosures relevant to the manuscript. W. Brück has received honoraria for lectures from Bayer Vital, Biogen Idec, Merck-Serono, Teva Pharma, Genzyme, Sanofi-Aventis, and Novartis and is a member of scientific advisory boards for Teva Pharma, Biogen Idec, Novartis, and Genzyme. Dr Brück serves on the editorial boards of Neuropathology and Applied Neurobiology, Multiple Sclerosis International, and Therapeutic Advances in Neurological Disorders. T. Chitnis has received personal compensation for advisory board/consulting from Biogen Idec, Merck-Serono, and Novartis and has received research support from Merck-Serono and Novartis Pharmaceuticals. She serves on pediatric clinical trial advisory boards for Genzyme-Sanofi and Novartis. Go to Neurology.org for full disclosures.

Received August 19, 2015. Accepted in final form February 19, 2016.

\section{REFERENCES}

1. Waldman A, Ghezzi A, Bar-Or A, Mikaeloff Y, Tardieu M, Banwell B. Multiple sclerosis in children: an update on clinical diagnosis, therapeutic strategies, and research. Lancet Neurol 2014;13:936-948.

2. Vargas-Lowy D, Chitnis T. Pathogenesis of pediatric multiple sclerosis. J Child Neurol 2012;27:1394-1407.

3. Moore CS, Cui QL, Warsi NM, et al. Direct and indirect effects of immune and central nervous system-resident cells on human oligodendrocyte progenitor cell differentiation. J Immunol 2015;194:761-772.

4. Popescu BF, Pirko I, Lucchinetti CF. Pathology of multiple sclerosis: where do we stand? Continuum (Minneap Minn) 2013;19:901-921.

5. Goldschmidt T, Antel J, König FB, Brück W, Kuhlmann T. Remyelination capacity of the MS brain decreases with disease chronicity. Neurology 2009;72:1914-1921.

6. Albert M, Antel J, Brück W, Stadelmann C. Extensive cortical remyelination in patients with chronic multiple sclerosis. Brain Pathol 2007;17:129-138.

7. Lucchinetti C, Bruck W, Parisi J, Scheithauer B, Rodriguez M, Lassmann $\mathrm{H}$. Heterogeneity of multiple sclerosis lesions: implications for the pathogenesis of demyelination. Ann Neurol 2000;47:707-717.

8. Kuhlmann T, Lingfeld G, Bitsch A, Schuchardt J, Bruck W. Acute axonal damage in multiple sclerosis is most extensive in early disease stages and decreases over time. Brain 2002;125:2202-2212.
9. Pfeifenbring S, Bunyan RF, Metz I, et al. Extensive acute axonal damage in pediatric multiple sclerosis lesions. Ann Neurol 2015;77:655-667.

10. Kuhlmann T, Lassmann H, Bruck W. Diagnosis of inflammatory demyelination in biopsy specimens: a practical approach. Acta Neuropathol 2008;115:275-287.

11. Young NP, Weinshenker BG, Parisi JE, et al. Perivenous demyelination: association with clinically defined acute disseminated encephalomyelitis and comparison with pathologically confirmed multiple sclerosis. Brain 2010; 133:333-348.

12. Probstel AK, Dornmair K, Bittner R, et al. Antibodies to MOG are transient in childhood acute disseminated encephalomyelitis. Neurology 2011;77:580-588.

13. Mayer MC, Breithaupt C, Reindl M, et al. Distinction and temporal stability of conformational epitopes on myelin oligodendrocyte glycoprotein recognized by patients with different inflammatory central nervous system diseases. J Immunol 2013;191:3594-3604.

14. Lennon VA, Wingerchuk DM, Kryzer TJ, et al. A serum autoantibody marker of neuromyelitis optica: distinction from multiple sclerosis. Lancet 2004;364:2106-2112.

15. Roemer SF, Parisi JE, Lennon VA, et al. Pattern-specific loss of aquaporin-4 immunoreactivity distinguishes neuromyelitis optica from multiple sclerosis. Brain 2007;130: 1194-1205.

16. Misu T, Hoftberger R, Fujihara K, et al. Presence of six different lesion types suggests diverse mechanisms of tissue injury in neuromyelitis optica. Acta Neuropathol 2013; 125:815-827.

17. Pohl D, Rostasy K, Reiber H, Hanefeld F. CSF characteristics in early-onset multiple sclerosis. Neurology 2004;63: 1966-1967.

18. Chabas D, Ness J, Belman A, et al. Younger children with MS have a distinct CSF inflammatory profile at disease onset. Neurology 2010;74:399-405.

19. Sinclair AJ, Wienholt L, Tantsis E, Brilot F, Dale RC. Clinical association of intrathecal and mirrored oligoclonal bands in paediatric neurology. Dev Med Child Neurol 2013;55:71-75.

20. Reiber H, Teut M, Pohl D, Rostasy KM, Hanefeld F. Paediatric and adult multiple sclerosis: age-related differences and time course of the neuroimmunological response in cerebrospinal fluid. Mult Scler 2009;15:1466-1480.

21. Baumann M, Sahin K, Lechner C, et al. Clinical and neuroradiological differences of paediatric acute disseminating encephalomyelitis with and without antibodies to the myelin oligodendrocyte glycoprotein. J Neurol Neurosurg Psychiatry 2015;86:265-272.

22. Heussinger N, Kontopantelis E, Gburek-Augustat J, et al. Oligoclonal bands predict multiple sclerosis in children with optic neuritis. Ann Neurol 2015;77:1076-1082.

23. Villar LM, Sadaba MC, Roldan E, et al. Intrathecal synthesis of oligoclonal IgM against myelin lipids predicts an aggressive disease course in MS. J Clin Invest 2005;115: 187-194.

24. Stauch C, Reiber H, Rauchenzauner M, et al. Intrathecal IgM synthesis in pediatric MS is not a negative prognostic marker of disease progression: quantitative versus qualitative IgM analysis. Mult Scler 2011;17:327-334.

25. Chitnis $\mathrm{T}$. The role of CD4 T cells in the pathogenesis of multiple sclerosis. Int Rev Neurobiol 2007;79:43-72.

26. Bar-Or A. The immunology of multiple sclerosis. Semin Neurol 2008;28:29-45. 
27. Dhaunchak AS, Becker C, Schulman H, et al. Implication of perturbed axoglial apparatus in early pediatric multiple sclerosis. Ann Neurol 2012;71:601-613.

28. Banwell B, Bar-Or A, Cheung R, et al. Abnormal T-cell reactivities in childhood inflammatory demyelinating disease and type 1 diabetes. Ann Neurol 2008;63:98-111.

29. Correale J, Tenembaum SN. Myelin basic protein and myelin oligodendrocyte glycoprotein T-cell repertoire in childhood and juvenile multiple sclerosis. Mult Scler 2006;12:412-420.

30. Vargas-Lowy D, Kivisakk P, Gandhi R, et al. Increased Th17 response to myelin peptides in pediatric MS. Clin Immunol 2013;146:176-184.

31. Balint B, Haas J, Schwarz A, et al. T-cell homeostasis in pediatric multiple sclerosis: old cells in young patients. Neurology 2013;81:784-792.

32. O'Connor KC, McLaughlin KA, De Jager PL, et al. Selfantigen tetramers discriminate between myelin autoantibodies to native or denatured protein. Nat Med 2007;13: 211-217.

33. Waters PJ, McKeon A, Leite MI, et al. Serologic diagnosis of NMO: a multicenter comparison of aquaporin-4-IgG assays. Neurology 2012;78:665-671; discussion 669.

34. Lennon VA, Kryzer TJ, Pittock SJ, Verkman AS, Hinson SR. IgG marker of optic-spinal multiple sclerosis binds to the aquaporin- 4 water channel. J Exp Med 2005; 202:473-477.

35. McLaughlin KA, Chitnis T, Newcombe J, et al. Agedependent $\mathrm{B}$ cell autoimmunity to a myelin surface antigen in pediatric multiple sclerosis. J Immunol 2009;183: 4067-4076.

36. Rostasy K, Mader S, Hennes EM, et al. Persisting myelin oligodendrocyte glycoprotein antibodies in aquaporin-4 antibody negative pediatric neuromyelitis optica. Mult Scler 2013;19:1052-1059.

37. Fernandez-Carbonell C, Vargas-Lowy D, Musallam A, et al. Clinical and MRI phenotype of children with MOG antibodies. Mult Scler 2016;22:174-184.

38. Dale RC, Tantsis EM, Merheb V, et al. Antibodies to MOG have a demyelination phenotype and affect oligodendrocyte cytoskeleton. Neurol Neuroimmunol Neuroinflamm 2014;1:e12. doi: http://dx.doi.org/10.1212/NXI. 0000000000000012.

39. Rostasy K, Mader S, Schanda K, et al. Anti-myelin oligodendrocyte glycoprotein antibodies in pediatric patients with optic neuritis. Arch Neurol 2012;69:752-756.

40. Huppke P, Rostasy K, Karenfort M, et al. Acute disseminated encephalomyelitis followed by recurrent or monophasic optic neuritis in pediatric patients. Mult Scler 2013 19:941-946.

41. Brilot F, Dale RC, Selter RC, et al. Antibodies to native myelin oligodendrocyte glycoprotein in children with inflammatory demyelinating central nervous system disease. Ann Neurol 2009;66:833-842.

42. Mader S, Gredler V, Schanda K, et al. Complement activating antibodies to myelin oligodendrocyte glycoprotein in neuromyelitis optica and related disorders. J Neuroinflammation 2011;8:184.

43. Spadaro M, Gerdes LA, Mayer MC, et al. Histopathology and clinical course of MOG-antibody-associated encephalomyelitis. Ann Clin Transl Neurol 2015;2:295-301.

44. Sato DK, Callegaro D, Lana-Peixoto MA, et al. Distinction between MOG antibody-positive and AQP4 antibody-positive NMO spectrum disorders. Neurology 2014;82:474-481.

45. Ketelslegers IA, Van Pelt DE, Bryde S, et al. Anti-MOG antibodies plead against MS diagnosis in an Acquired Demyelinating Syndromes cohort. Mult Scler 2015;21: 1513-1520.

46. Srivastava R, Aslam M, Kalluri SR, et al. Potassium channel KIR4.1 as an immune target in multiple sclerosis. N Engl J Med 2012;367:115-123.

47. Kraus V, Srivastava R, Kalluri SR, et al. Potassium channel KIR4.1-specific antibodies in children with acquired demyelinating CNS disease. Neurology 2014;82:470-473.

48. Nerrant E, Salsac C, Charif M, et al. Lack of confirmation of anti-inward rectifying potassium channel 4.1 antibodies as reliable markers of multiple sclerosis. Mult Scler 2014; 20:1699-1703.

49. Brickshawana A, Hinson SR, Romero MF, et al. Investigation of the KIR4.1 potassium channel as a putative antigen in patients with multiple sclerosis: a comparative study. Lancet Neurol 2014;13:795-806.

50. Van Haren K, Tomooka BH, Kidd BA, et al. Serum autoantibodies to myelin peptides distinguish acute disseminated encephalomyelitis from relapsing-remitting multiple sclerosis. Mult Scler 2013;19:1726-1733.

51. Quintana FJ, Patel B, Yeste A, et al. Epitope spreading as an early pathogenic event in pediatric multiple sclerosis. Neurology 2014;83:2219-2226.

52. Pouwels PJ, Vanderver A, Bernard G, et al. Hypomyelinating leukodystrophies: translational research progress and prospects. Ann Neurol 2014;76:5-19.

53. Brown RA, Narayanan S, Banwell B, Arnold DL; Canadian Pediatric Demyelinating Disease Network. Magnetization transfer ratio recovery in new lesions decreases during adolescence in pediatric-onset multiple sclerosis patients. Neuroimage Clin 2014;6:237-242.

54. Aubert-Broche B, Fonov V, Narayanan S, et al. Onset of multiple sclerosis before adulthood leads to failure of ageexpected brain growth. Neurology 2014;83:2140-2146.

55. Kerbrat A, Aubert-Broche B, Fonov V, et al. Reduced head and brain size for age and disproportionately smaller thalami in child-onset MS. Neurology 2012;78:194-201. 


\title{
Neurology
}

\section{Immunopathophysiology of pediatric CNS inflammatory demyelinating diseases}

\author{
Amit Bar-Or, Rogier Q. Hintzen, Russell C. Dale, et al.
}

Neurology 2016;87;S12-S19

DOI 10.1212/WNL.0000000000002821

This information is current as of August 29, 2016

\section{Updated Information \&} Services

References

Permissions \& Licensing

Reprints including high resolution figures, can be found at: http://n.neurology.org/content/87/9_Supplement_2/S12.full

This article cites 55 articles, 5 of which you can access for free at: http://n.neurology.org/content/87/9_Supplement_2/S12.full\#ref-list-1

Information about reproducing this article in parts (figures,tables) or in its entirety can be found online at:

http://www.neurology.org/about/about_the_journal\#permissions

Information about ordering reprints can be found online:

http://n.neurology.org/subscribers/advertise

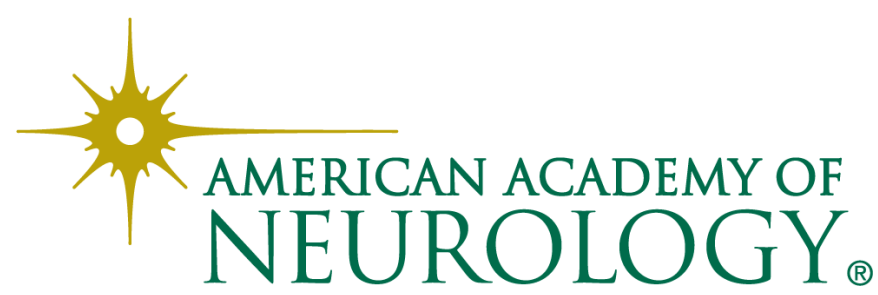

